\title{
A CLASS OF WILD CLOSED CURVES THAT SPAN ORIENTABLE SURFACES ${ }^{1}$
}

\author{
O. G. HARROLD, JR.
}

\begin{abstract}
A classical result in the topology of manifolds asserts that every polygonal closed curve in three-space bounds an orientable surface. In this note we relax the condition that the curve be locally tame and obtain a partial generalization.
\end{abstract}

THEOREM 1. If a simple closed curve $k$ is a boundary component of an annulus $A$ that is untwisted and unknotted and if $k=\bigcap_{i=1}^{\infty} T_{i}$, where $T_{i}$ is a solid torus, then $k$ bounds a disk.

The author wants to express his appreciation to Wolfgang Heil and Shin'ichi Kinoshita for suggesting improvements in the original manuscript.

Preliminaries. Since we are not in the $P L$ category it is necessary to explain what is meant by saying $A$ is untwisted and unknotted. By the Bing Approximation Theorem [2], we may take $A$ locally polyhedral $\bmod k$ and each $\dot{T}_{i}$ (=boundary of $T_{i}$ ) polyhedral. If $k^{\prime}$ is a polygonal nonbounding curve on $A \backslash k$ near $k$, then $k^{\prime}$ and the other boundary component $l$ of $A$ determines a polyhedral annulus to which the criteria of Kyle applies [6]. In essence it is assumed these annuli are untwisted and unknotted.

Statement I. Hypothesis as in Theorem 1; conclusion: $k$ bounds an orientable surface. (The conclusion that $k$ bounds an orientable surface holds even if $A$ is knotted.)

Proof of Statement I. The simple closed curves $k$ and $l$ are the boundary components of $A$. Since $k=\bigcap_{1}^{\infty} T_{i}$, there is an integer $N$ such that $\dot{T}_{N}$ separates $k$ and $l$. By general position considerations we assume

$$
A \cap \dot{T}_{N}=(A \backslash k) \cap \dot{T}_{N}=s_{1} \cup \cdots \cup s_{m},
$$

is a nonempty collection of pairwise disjoint simple closed curves. By a familiar disk replacement argument, no $s_{i}$ that is $\sim 0$ on both $\dot{T}_{N}$ and $A$ need occur. If $s_{i} \nsim 0$ on $A$ but $s_{i} \sim 0$ on $\dot{T}_{N}$ we easily find the desired disk

Received by the editors July $26,1972$.

AMS (MOS) subject classifications (1970). Primary 55A30, 57A10; Secondary 55A25.

Key words and phrases. Wild closed curves, untwisted annuli, solid tori.

${ }^{1}$ Research supported in part by NSF GP-19964.

(c) American Mathematical Society 1973 
by replacing a circular ring on $A$ (not containing $k$ ) by a disk of $\dot{T}_{N}$. The problem is thus reduced to the case no $s_{i} \sim 0$ on $\dot{T}_{N}$. By the fact that $s_{1}, \cdots, s_{m}$ are disjoint, all $s_{i}$ 's are longitudes or nonlongitudes.

Consider the case all $s_{i}$ 's are nonlongitudes. Then each $s_{i}$ links $k$. (Although $k$ is not a polyhedral core of $T_{N}$, there is a polyhedral core as close to $k$ as we like.) Now $s_{i} \sim l$ on $A \backslash k$. Hence $l$ links $k$ and hence $l$ links any sufficiently good approximation to $k$. But the annulus determined by $l$ and such an approximation to $k$ is untwisted by hypothesis. This is impossible, and each $s_{i}$ must be a longitude. By definition of longitude, there is a surface $B$ such that $s_{i}=\partial B, B \subset S^{3} \backslash T_{N}$. If $s_{i}$ separates $A$ into $A^{\prime}$ and $A^{\prime \prime}$, we may suppose $A^{\prime}$ contains $k$ and $A^{\prime} \cap\left(S^{3} \mid T_{N}\right)=\square$. Then $B \cup A^{\prime}$ is the desired surface. This proves Statement I.

It remains to show that the surface may be taken to be a disk under the full hypothesis of Theorem 1 regarding $A$. The tori $T_{1}, T_{2}, \cdots$ by Theorem 2 below may be assumed concentric (see [3] or [5] for definition of concentric). Let us assume the tori are concentric for the moment and finish the proof that $k$ bounds a disk.

We must show some $T_{N}$ (say $T_{1}$ ) is unknotted. Since $T_{1}$ and $T_{2}$ are concentric, $\mathrm{Cl}\left(T_{1} \mid T_{2}\right)$ contains an annulus $Z$ with one boundary component on $\dot{T}_{1}$ and one on $\dot{T}_{2}$. Since $l$ and $k$ are exterior to $\dot{T}_{1}$ and interior to $\dot{T}_{2}$, respectively, a $Z$ can be found that is a subset of $A$. The fact that $Z$ is unknotted and untwisted means its boundary components can approximate a polygonal core of $T_{1}$ arbitrarily closely and therefore $T_{1}$ must be unknotted. Thus the surface $B$ can be taken to be a disk and $k$ bounds a (possibly wild) disk. (See examples of Alford [1] and Gillman [4].)

THEOREM 2. If $k$ is a boundary component of an annulus $A$ that is untwisted and unknotted and if $k=\bigcap_{1}^{\infty} T_{i}$, where $T_{i}$ is a solid torus, then for some subsequence $i_{1}, i_{2}, \cdots$ of the integers $T_{i_{k+1}}$ is concentric to $T_{i_{k}}$.

Remarks. As mentioned above each $T_{i}$ may be assumed polyhedral. If solid tori $B$ and $B^{*}$ are such that $B \subset$ int $B^{*}$, then $B$ and $B^{*}$ are concentric iff $\mathrm{Cl}\left(B^{*} \mid B\right)=S^{1} \times S^{1} \times[0,1]$. An integer $O\left(B^{*}, B\right)$ is defined by Schubert [8] for any such pair of solid tori. Since $k=\bigcap T_{i}$, and $A$ is untwisted and unknotted we are concerned only with the cases $O\left(T_{i}, T_{j}\right)= \pm 1, i \neq j$. By a criterion of Edwards [3], concentricity of $B$ and $B^{*}$ in the presence of $O\left(B, B^{*}\right)= \pm 1$ is equivalent to the equivalence of the knot-types of the center lines of $B$ and $B^{*}$.

Proof OF TheOREM 2. Suppose, on the contrary, there is a subsequence $i_{1}, i_{2}, \cdots$ such that $T_{i_{k+1}}$ is knotted in $T_{i_{k}}, k=1,2, \cdots$. By passing to a subsequence, we may suppose $i_{k}=k$. Thus $T_{2}$ is knotted in $T_{1}, T_{3}$ in $T_{2}$, $\cdots$. Let $T_{k}$ have a polygonal center line $l_{k}$. Each $l_{k}$ has a unique decomposition (apart from the order) into prime nontrivial knots. There is a 
subannulus of $A$ with boundary components $l_{k}$ and $l_{k+j}$. Now the subannulus of $A$ with these boundary components is untwisted and unknotted, by hypothesis. Therefore the total curvature of each $l_{k}$ and $l_{k+j}$ is $2 \pi$. But it is well known that a polygonal knotted curve, i.e. $\left(l_{k}\right)$, has a total curvature $\geqq 4 \pi[7]$.

\section{REFERENCES}

1. W. R. Alford, Some "nice" wild 2-spheres in $E^{3}$, Topology of 3-Manifolds and Related Topics (Proc. The Univ. of Georgia Inst., 1961), Prentice-Hall, Englewood Cliffs, N.J., 1962, pp. 29-33. MR 25 \#4504.

2. R. H. Bing, Approximating surfaces with polyhedral ones, Ann. of Math. (2) 65 (1957), 456-483. MR 19, 300.

3. C. H. Edwards, Jr., Concentric solid tori in the 3-sphere, Trans. Amer. Math. Soc. 102 (1962), 1-17. MR 25 \#3514.

4. D. S. Gillman, Sequentially 1-ULC tori, Trans. Amer. Math. Soc. 111 (1964), 449-456. MR 28 \#5433.

5. O. G. Harrold, Jr., H. C. Griffith and E. E. Posey, A characterization of tame curves in three-space, Trans. Amer. Math. Soc. 79 (1955), 12-34. MR 19, 972.

6. R. H. Kyle, Embeddings of Möbius bands in 3-dimensional space, Proc. Roy. Irish Acad. Sect. A 57 (1955), 131-136. MR 19, 976.

7. J. W. Milnor, On the total curvature of knots, Ann. of Math. (2) 52 (1950), 248-257. MR 12, 273.

8. H. Schubert, Knoten und Vollringe, Acta Math. 90 (1953), 131-286. MR 17, 291.

Department of Mathematics, Florida State University, Tallahassee, Florida 32306 